\title{
THE GIANT PLANETS AND THEIR SATELLITES: REPORT ON THE COSPAR SYMPOSIUM, OTTAWA, CANADA, MAY 18-21, 1982
}

\author{
M. G. Kivelson ${ }^{1}$, K. W. Behannon 2 , T. E. Cravens ${ }^{3}$ \\ I. de Pater ${ }^{4}$, T. V. Johnson ${ }^{5}$, H. Masursky6, D. L. Matson ${ }^{5}$, \\ D. J. Southwood 7 and V. M. Vasyliunas8 \\ 'University of California, Los Angeles, CA 90024, U.S.A. \\ 2NASA Goddard Space Flight Center, Greenbelt, MD 20771, U.S.A. \\ ${ }^{3}$ University of Michigan, Ann Arbor, MI 48109, U.S.A. \\ "University of Arizona, Tucson, AZ 85721, U.S.A. \\ ${ }^{5}$ California Institute of Technology, Pasadena, CA 91103, U.S.A. \\ ${ }^{6}$ U.S. Geological Survey, Flagstaff, AZ 86001, U.S.A. \\ 7 Imperial College, London, U.K. \\ ${ }^{8}$ Max-Planck-Institut für Aeronomie, Katlenburg-Lindau, F.R.G.
}

\section{ABSTRACT}

A Symposium on the Giant Planets and Their Satellites was presented in conjunction with the Twenty-fourth Plenary Meeting of the Committee on Space Research. This paper summarizes the talks presented and places the remaining papers of this volume in context.

INTRODUCTION

The giant planets and their satellites have attracted the interest of scientists over the centuries. Galileo's observations of the four large satellites of Jupiter provided the analogy he required to persuade himself of the validity of Kepler's laws and of their relevance to planetary motion about the sun. Today, too, Jupiter, Saturn and their satellites provide laboratories in which processes important in terrestrial systems or even in exotic astrophysical systems are occurring in a range of parameter space (spatial scale, rotation rate, etc.) nowhere else accessible.

The in situ measurements provided by the Pioneers 10 and 11 and Voyagers 1 and 2 flybys of the outer planets have revealed unexpected features of the planet-satellite systems. Atmospheric circulation patterns in the rapidly rotating jovian atmosphere, heated from below as we11 as from above, have led to new ideas about the transfer between eddies and largescale flow. Radio-frequency emissions observed near Jupiter and Saturn are providing insight into generation mechanisms important in the terrestrial magnetosphere. Waves and particles in Jupiter's environment are modulated at the planetary rotation period, and this pulsar-like behavior provides insight into mechanisms important in inaccessible objects. Satellite surfaces show evidence of tectonic processes, originally unanticipated in such small bodies. The importance of tidal heating for satellite systems in resonant orbits was dramatically demonstrated when active volcanoes were found on Io. Rings, plumes, tori have further illustrated the inevitability of encountering the unexpected.

The rich lode of new data garnered by spacecraft flybys of the outer planets has engendered an explosion of interpretive studies. Spring of 1982 proved to be a good time to take stock of what has been learned and where critical questions remain unanswered. Ottawa, Canada, provided an agreeable locale for a four-day (May 18-21, 1982) Symposium on the Giant Planets and Their Satellites presented as part of the XXIV COSPAR (Committee on Space Research) Plenary Meeting. 1/ The sessions were well attended despite constraints on travel budgets and competition from other spring 1982 meetings directed at similar audiences.

The program organization emphasized scientific problems and brought together speakers familiar with many aspects of recent work. For example, in the session on Io's plasma torus, spectroscopists discussed data obtained both from the ground and from space, and plasma theorists considered the implications of standard as well as most innovative physical arguments for data interpretation. Fully consistent descriptions of ion sources, of energy

1/ Other organizations sponsoring the Symposium were the International Astronomical Union (IAU), the International Association on Meteorology and Atmospheric Physics (IAMAP of IUGG), the International Union of Theoretical and Applied Mechanics (IUTAM) and the International Union of Radio Science (URSI). 
exchange between hot, newly ionized fons and cool electrons, and of the partitioning of ions into different charge states have proved elusive. Lively exchanges, predictably, characterized the discussions of the presentations dealing with these problems. Other equally refractory issues emerged in discussions of rings, of planetary radio astronomy, and of interiors, surfaces and atmospheres.

The descriptions of the individual sessions that follow provide a sense of the diverse interests which come together in the study of planetary systems once in situ spacecraft data are widely available for study. That situation exists for Jupiter, Saturn, and their satel1ites. Data from Pioneers 10 and 11 and from Voyagers 1 and 2 have not only brought Saturn's rings to the coffee tables of the world and made the surface of Io as familiar as the dunes of the Sahara, but have attracted scientists from different disciplines to their analysis. Uranus and Neptune remain the domain of astronomers (only one paper considered these other giant planets), but with Voyager 2 now targeted at Uranus, the situation should be different at some future COSPAR conference.

In the detailed description of the Symposium which follows, asterisks indicate participants whose contributions were submitted for publication and appear elsewhere in this volume.

\section{PLANETARY AND SATELLITE INTERIORS AND SURFACES}

E. C. Stone (California Institute of Technology, Pasadena, CA, USA), a last-minute substitute for B. A. Smith (University of Arizona, Tucson, AZ, USA), opened the Symposium with a review of results based on Voyagers 1 and 2 imaging of Jupiter and Saturn. The atmospheric circulation patterns and compositions differ between the two planetary atmospheres; belts and zones are prominent on both, but the cloud velocities are higher on Saturn and the variation of velocity with latitude is smoother, with the equatorial velocities almost three times higher on Saturn than on Jupiter. Violent turbulence at the boundaries between adjacent belts and zones is notable on Jupiter. The internally generated heat exceeds the heat absorbed from the Sun by a factor of two on Saturn.

Saturn's ring system is more complex than Jupiter's. Major gaps in rings occur at orbits satisfying conditions of resonance with the large satellites, but additional spiral patterns of "ringlets" and "gaplets" are observed. The finest detail was provided by the photopolarimeter observations of a stellar occultation. Impressive evidence of spiral density waves in the rings was presented. The narrow rings (e.g. the F-ring) are shepherded by small satellites. The "spokes" in the B-ring are now being interpreted as evidence of intermittent levitation of small particles above the ring plane by electrostatic or electromagnetic forces.

Properties of the galilean sateliites of Jupiter vary systematically from the innermost, Io, with a surface wholly covered by internally generated deposits, to the outermost, Callisto, with a surface everywhere scarred by impact craters. The surfaces of the saturnian satellites vary from Enceladus, with a surface strongly modiffed by internal activity, to Rhea, with its highly cratered surface. New, small satellites are still being discovered by reexamination of spacecraft images and ground-based photographs.

S. J. Peale (University of California, Santa Barbara, CA, USA) discussed the consequences of the locking of the galilean satellites Io, Europa and Ganymede (referred to as satellites 1 , 2 , and 3 , respectively) in stable orbital resonance. The mean angular velocities, $\mathrm{n}_{1}$, are related by $2 n_{1}-3 n_{2}+2 n_{3}=0$. If the resonance is primordial, the proximity of Io to Jupiter puts a lower bound on the mean dissipation function Qj of Jupiter of Qj $>6 \times 10^{4}$.

The requirement that conjunctions of the satellites occur when Io is near its orbit pericenter and Europa near either pericenter or apocenter force eccentricities $\left(e_{i}\right)$ into the orbits of these satellites. The eccentricities, in turn, lead to dissipation caused by now-varying tides raised by Jupiter. This dissipation tends to reduce $e_{i}$, whereas further expansion of the orbits by the tides that Io raises on Jupiter tends to increase $e_{i}$. The two effects balance at equilibrium values of $e_{1}$ which thereafter remain nearly constant as expansion of the orbit continues. Interpretation of the currently observed value of Io's orbital eccentricity, $\mathrm{e}_{1}=.0041$, as an equilibrium value leads to a ratio of tidal disstpation in Io to that in Jupiter of $k_{1} / Q_{1} \ll 900 \mathrm{kj} / Q_{j}$, where the $k^{\prime} s$ are Love numbers. An upper bound of $Q_{1}$, deduced from a requirement that tidal dissipation exceed heating from radioactive decay, places an upper bound on $Q_{j}$ of $2 \times 10^{6}$. This upper bound is much less than some theoretica1 estimates of $Q_{j}$, whereas direct measurement of heat flow from Io lowers $Q_{1}$ so drastically as to push $Q_{j}$ below the least lower bound. Possible resolutions to this paradox require further study.

D. L. Matson (Jet Propulsion Laboratory, Pasadena, CA, USA) observed that the heat flow on Io is currently about $2 \mathrm{~W} \mathrm{~m}^{-2}$ based on spacecraft and ground-based observations. This global heat value is comparable to that from active geothermal areas on Earth. Matson believes that the origin of the heat flow is tidal dissipation, as originally suggested by Peale, Cassen and Reynolds. Peale thinks it may not be possible to produce this high a value as a steady- 
state condition on long time scales. Either activity on Io is episodic or the geophysical parameters are still not well known.

Serving as pinch-hitter for E. M. Shoemaker, H. Masursky (U. S. Geological Survey, Flagstaff, $A Z$, USA) summarized ideas regarding the thermal evolution of Ganymede and Callisto. (In a later session, Shoemaker made his own presentation as mentioned below.) Thermal histories of these satellites can be inferred from the viscous relaxation of craters on their surfaces. The thermal gradient on Ganymede decayed very rapidly near the end of heavy bombardment at about $4 \mathrm{~Gy}$. The $1 \mathrm{i}$ thosphere thickened from about $10 \mathrm{~km}$ at an early epoch to about $100 \mathrm{~km}$ at the time that the large Gilgamesh basin formed. Grooved terrain resulted from slight crustal extension faulting, eruption of aqueous fluids and shallow convection in an ice asthenosphere. Callisto had a similar cooling history but cooled about 200 My earlier and no grooved terrain formed.

Masursky's own paper considered the great variation among outer planetary satellites in the degree of resurfacing of cratered terrain by internal activity. Such resurfacing ranges from complete on Io to partial on Enceladus and Dione to little on Callisto. Enceladus has the most complex history and its surface includes both preserved ancient cratered terrain and smooth plains with no discernible craters. Different types of tectonic activity break the surface, the energy probably being provided by tidal effects. Additional effects on the satellites may be related to the electrostatic or electromagnetic effects that produce the ring spokes.

The significant effect of tides on the satellites of the outer planets may indicate that the lack of a moon may be critical to the differences in tectonic/volcanic style between Venus and Earth. Other important differences are Venus' slow rotation and slightly smaller radius and density.

E. M. Parmentier (Brown University, Providence, RI, USA) discussed tectonic features on icy satellites. The systems of dark lineaments on the nearly crater-free Europa may be fractures formed in response to tidal stresses that place constraints on the orbital evolution. In contrast, the bright terrain on Ganymede results when rifts in old dark terrain are filled by water-rich magma following internal heating or differentiation. On Callisto, multi-ringed structures created by large impacts are the only "tectonic" features recognized. The smaller saturnian satellite, Enceladus, shows features similar to Ganymede.

D. Gautier (Observatoire de Meudon, Meudon, France) discussed the helium, deuterium and carbon abundances in Jupiter and Saturn and their consequences for the theory of solar system formation. Voyager infrared observations indicate the $\mathrm{C} / \mathrm{H}$ ratio 1 s enriched in Jupiter compared to solar values by a factor of $2.07 \pm 0.24$. The ground-based measurements also indicate an enrichment in carbon. These results favor the theory of homogeneous formation of the giant planets by the accretion of grains of refractory materials and ices embedded in the primordial solar nebula. The relative helium abundance in Saturn's outer atmosphere is half that found at Jupiter and in the solar atmosphere. The reduction is thought to occur because the differentiation of helium from hydrogen at high pressures in the interior of the planet concentrates helium at great depths within Saturn. Such depletion does not seem to exist on Jupiter, which allows us to infer the helium abundance in the primitive nebula; the upper limit is equal to 24 percent by mass. The primitive helium is produced in the big bang. Jovian deuterium $\left(\mathrm{D} / \mathrm{H}=3.6 \pm 1.0 \times 10^{-5}\right)$ leads to an estimate of the upper limit of the primordial deuterium and to a limit on the baryon density. Gautier's talk ended on a cosmological theme with a discussion of the implications of the jovian isotopic abundance ratios as tests of the standard "Big-Bang" theory.

\section{SATELLITE TORI AND PLUMES}

The session on satellite tori and plumes consisted of six invited papers and one contributed paper. Perhaps the most challenging paper to western ears was the one contributed by $A$. A. Galeev* (USSR Academy of Sciences, Moscow, USSR) and I. Chabibrachmanov of the USSR which looked at the ionization of neutral atmospheres. The paper used plasma instability theory to provide a firm basis for the "critical velocity" ionization phenomenon first suggested by Alfvén in 1942. The detailed theory yields a critical velocity six times that of Alfvén's original estimate. At Titan, the saturnian magnetospheric corotation speed is above the critical velocity for ionization of the neutral hydrogen cloud, but the slowing up of flow in the satellite's vicinity may inhibit the process. At Io, the plasma speed exceeds both the critical velocity for sulfur and oxygen. The instability sustains plasma waves that generate a suprathermal electron population whose wave emission spectrum could be seen in the Voyager plasma wave data recorded in the torus.

The ionization and subsequent redistribution of energy gained in ion pickup to electrons and ultimately to UV emission was a general concern in several papers presented. In contrast to Galeev's presentation, R. A. Smith (Science Applications, Inc., McLean, VA 22102, USA) had derived a model in which, by detailed treatment of the nonequilibrium plasma phase-space- 
distributions, he concluded that collisions could in fact explain the energy redistribution and emission in spite of original prejudices to the contrary.

R. A. Brown (University of Arizona, Tucson, AZ, USA) had opened the session with a discussion of ground-based photometric studies of relative ion concentrations in the Io torus. He presented evidence of a 5\% departure from corotation in the torus based on measured Doppler shifts. Further Doppler analysis was used to display the non-Maxwellian nature of the $s^{+}$ population. The mean energy deduced from a detalled knowledge of the distribution is 60 eV in contrast with an FWHM estimate of $10 \mathrm{eV}$. He also put considerable emphasis on the importance of charge exchange in various phenomena, fast neutral sodium ejection, the apparent lack of equilibrium between $0^{2+}$ and the electron population, and discrepancies between $\mathrm{s}^{2+}$ and $\mathrm{S}^{+}$populations in thermal and spatial distribution.

W. H. Smyth (Atmospheric and Environmental Research, Inc., Cambridge, MA, USA) considered the neutral gas clouds surrounding and extending well beyond the gravitational control of satellites in both jovian and saturnian systems. Io has sodium, potassium and oxygen clouds. Titan has a hydrogen cloud. Conceivably Tethys or Dione could have a neutral cloud made up from dissociated water products. Io could have a neutral sulfur cloud. Predicted intensity is some $10 \%$ down on the $8 \mathrm{R}$ of the oxygen cloud. Electron impact ionization lifetimes vary from species to species, and cloud shapes and extents vary. East-west asymetry is introduced by solar radiation pressure and north-south asymmetry by the oscillation of the Io plasma with respect to the neutral cloud.

D. F. Strobel (Nava1 Research Laboratory, Washington, D.C., USA) reviewed UV observations of the Io plasma torus during Voyager encounters. Strong emission lines of $\mathrm{s}^{2+}, \mathrm{s}^{3+}, 0^{+}$, and $\mathrm{O}^{2+}$ were detected. Earlier modeling has been thrown into confusion by revised colliston cross-sections. (An error in the standard estimates of spectral frequencies for SII emissions was also reported.) The Io torus is some $35 \%$ brighter on the dusk side of Jupiter. There is an Io signature of about $20 \%$. It was suggested the $35 \%$ excess could be a residual solar wind-associated effect. Strobel also emphasized the role of charge exchange both as a source and sink of ions in the inner torus.

R. M. Thorne (University of California, Los Angeles, CA, USA) considered the problem of the torus-related aurora. He pointed out that the obvious source for the auroral primaries was not electrons, but energetic ions which are depleted in the Io vicinity. He reviewed the theory of stable trapping of radiation belt particles. The flux is near the stable limit in the inner magnetosphere but is maintained above it by rapid radial transport. He also reviewed the observed wave spectra. Ions are precipitated by electromagnetic ion cyclotron waves. Protons above $10 \mathrm{keV}$ can be precipitated by such waves. The rapid removal of such ions can be fit with accepted radial diffusion theories, but substantial changes in the diffusion coefficient appear to have to be allowed for. The data suggest the coefficient is independent of energy.

A. Nishida (University of Tokyo, Tokyo, Japan) and Y. Watanabe described a mechanism for jovian ionospheric heating driven by the solar wind. The combination of substantial radial magnetopause motions and high rotation speed of the jovian magnetosphere mean substantial torques need be exerted between ionosphere and magnetospheric plasma after magnetopause compressions. The stresses are taken up by the magnetic field. Heating rates of $10^{13} \mathrm{~W}$ can be achieved with peak local rates occurring on high-latitude $\left(76^{\circ}\right)$ flux tubes and a lowerlatitude peak where mass density is greatest. Similar arguments have been applied by Nishida and colleagues to the problem of spin-up of outward-diffusing plasma from a source in the Io torus.

\section{AERONOMY AND DYNAMICS}

Two sessions on aeronomy and dynamics, as well as composition and evolution of the planetary atmospheres, followed.

S. K. Atreya (University of Michigan, Ann Arbor, MI, USA) showed Voyager UV, radio and IR data relevant to the region in the upper atmospheres at pressures $<1 \mathrm{mb}$. The exospheric temperature of Juptter is $1100 \pm 200 \mathrm{~K}$, of Saturn $600-800 \mathrm{~K}$. The vertical mixing on Saturn is much more efficient than on Jupiter: the eddy diffusion coefficient at the homopause on Jupiter is $10^{6} \mathrm{~cm}^{2} / \mathrm{sec}$, on Saturn $10^{8} \mathrm{~cm}^{2} / \mathrm{sec}$. Both values were derived from observations of methane as well as from Lyman alpha data. The topside ionospheric structure for both planets shows a peak electron density, well below the calculated values, which lies higher in the atmosphere than expected. He explored the possibility of an additional sink for protons by vibrationally excited $\mathrm{H}_{2}$. In the equatorial regions an energy input of $0.3 \mathrm{erg} / \mathrm{cm}^{2}-\mathrm{sec}$ on Jupiter and of $0.05 \mathrm{erg} / \mathrm{cm}^{2}-\mathrm{sec}$ on Saturn has been inferred from observations of the $\mathrm{H}_{2}$-Lyman and Werner bands, and 5 to $10 \mathrm{erg} / \mathrm{cm}^{2}-\mathrm{sec}$ and $0.5 \mathrm{erg} / \mathrm{cm}^{2}-\mathrm{sec}$, respectively, at higher latitudes. For Jupiter, thermospheric heating can be satisfactorily accounted for by energetic electrons; how to explain the energy budget on Saturn, however. remains a puzzle. 
A. P. Ingersol1 (California Institute of Technology, Pasadena, CA, USA) presented results on the atmospheric dynamics of Jupiter and Saturn, with particular emphasis on the long-life and banded structure of large-scale cloud features at pressure levels around 1 bar. He questioned whether there is a shallow weather layer to which the zonal winds are confined or whether these winds reflect fluid motions that extend through the planet on differentially rotating cylinders; he argued in favor of the deep atmospheric circulation. Measurements of Saturn's mainly eastward zonal wind profile and upper bounds on the latitudinal temperature gradient imply that the level of uniform rotation is at pressures well in excess of 1000 bars. If the winds extend through the planet on differentially rotating cylinders, one would expect symmetry between the northern and southern hemispheres at the latitudes where the cylinders emerge into the atmosphere. The symmetry is present at low latitudes, but breaks down for Jupiter above $45^{\circ}$ and for Saturn above $65^{\circ}$. In both cases, the breakdown of symmetry can be explained by a different size of the planets' metallic cores. He further showed that the small-scale eddies carry momentum into the mean flow and, if the zonal winds extend deep into the atmosphere, the very long-lived ovals in the transition zones between $\mathrm{E}$ and $\mathrm{W}$ jets correspond to features obtained in numerical models and laboratory experiments.

G. E. Hunt (Imperial College, London, U.K.) presented a paper on the composition and abundance ratios in the atmosphere of Jupiter and Saturn and on tropospheric meteorology. The main constituents in Jupiter's atmosphere are $\mathrm{H}_{2}, \mathrm{NH}_{3}$, and $\mathrm{CH}_{4}$. On Saturn, $\mathrm{NH}_{3}$ was not a dominant feature; instead, $\mathrm{PH}_{3}$ dominates the spectrum, which indicates a strong upward atmospheric motion. Further emission lines of $\mathrm{C}_{2} \mathrm{H}_{2}$ and $\mathrm{C}_{2} \mathrm{H}_{6}$ are dominant features in Saturn's spectrum. Unlike Jupiter, Saturn shows no longitudina1 structures, and a temperature gradient between the poles and the equator was measured. Unlike Ingersoll, Hunt remarked on a surprisingly close similarity among the weather systems on Earth, Jupiter, and Saturn, although the features and driving mechanisms are entirely different on the three planets.

J. J. Caldwel1 (University of New York at Stony Brook, NY, USA) showed some remarkable auroral effects, measured in the $\mathrm{CH}_{4}$ band at $8 \mu$. There is a strong polar brightening at longitudes near $200^{\circ}$ ( $\mathrm{N}$. pole) and $20^{\circ}$ (S. pole), where the north polar region (zenographic latitude of $70^{\circ}$ ) becomes relatively brighter than the south polar region. The brightening feature is extended in the $\mathrm{E}-\mathrm{W}$ direction and is very close to the $1 \mathrm{imb}$, closer than the auroral zone or the magnetic mapping of the Io plasma torus. Since a similar brightening occurs in all molecules, he argued that it must be a thermal effect, caused by high energy (10-20 MeV) electron impact.

Papers on Saturn and Titan dominated the second session on aeronomy and dynamics, although some papers on the other outer planets and satellites were also presented.

P. Gierasch (Corne11 University, Ithaca, NY, USA) and coworkers presented infrared observations of Saturn and Titan by Voyager. Measurements of the infrared emission of Saturn enabled them to determine that the ratio of the thermal emission to the absorbed solar radiation is $1.79 \pm 0.1$, which is still higher than the value of $1.67 \pm 0.09$ found for Jupiter and somewhat less than was previously thought to be the case for Saturn. Gierasch also reported that stratospheric temperatures for Titan have significant latitudinal gradients. Because the rotation rate of Titan is sma11, they suggested that the winds resulting from the temperature-related pressure gradients must be cyclostrophic rather than geostrophic. Titan is the second-known example in the solar system of strong global-scale cyclostrophic winds, the other example being Venus.

New information on Saturn and its rings was presented by I. de Pater* (University of Arizona, Tucson, AZ, USA) and J. R. Dickel who reported on results of high-resolution radio observations at 1,2 and $6 \mathrm{~cm}$ using the VLA (very large array). Limb darkening in the N-S directions was less than anticipated on the basis of model calculations. This may imply reduced ammonia abundance near the polar regions. D. M. Hunten (University of Arizona, Tucson, AZ, USA) described the atmosphere of Titan: predominantly nitrogen, but containing methane, possibly argon, and many photochemical products including the ubiquitous brown smog which hides the surface and lower atmosphere. He discussed two scenarios for the evolution of a nitrogen atmosphere: outgassing and photolysis of ammonia, and capture of nitrogen directly from the proto-saturnian nebula. He argued in favor of the former mechanism, although he did not rule out the latter entirely. J. J. Caldwe11 (State University of New York at Stony Brook, NY, USA) discussed IUE (International U1traviolet Explorer) observations of Saturn, Uranus, and Neptune and suggested that about $6 \times 10^{-3} \mathrm{~cm}-\mathrm{atm}$ of $\mathrm{H}_{2} \mathrm{O}$ might be present in the atmosphere of Saturn. R. A. West* (University of Colorado, Boulder, CO, USA) and coworkers investigated the latitudinal distribution of UV absorbers in the atmosphere of Saturn using Voyager photopolarimeter data. S. Kumar (University of Southern California, Los Angeles, CA, USA) reviewed the current status of theory and observation of the atmosphere and ionosphere of Io. Currently, none of the models for Io's atmosphere is compatible with all the avallable observations. In particular, Kumar summarized the arguments for and against the thin and thick $\mathrm{SO}_{2}$ atmosphere models of Io. He noted that the role of volcanic outgassing is not understood. L. F. Trafton* (University of Texas at Austin, Austin, TX, USA) presented Raman scattering measurements of Saturn's equatorial haze layer which suggest that the aerosols behave quite differently in the equatorial region than at other latitudes. 
T. Owens (State University of New York at Stony Brook, NY, USA) compared Uranus and Neptune and pointed out that although these planets are commonly thought of as twins, it has become apparent that they are dissimilar in many important respects. The axis of rotation of Uranus is almost parallel to the plane of the ecliptic, unlike Neptune (or any other planet in the solar system). Uranus has rings, whereas Neptune does not have any detectable ring system. 2/ Uranus has no large satellites and none with retrograde orbits, unlike Neptune. And, unlike all the other major planets, Uranus has neither a detectable internal heat source nor detectable tropospheric clouds.

\section{INTERACTION OF MAGNETOSPHERIC PLASMA WITH RINGS AND SATELLITES}

A session on magnetospheric interactions followed. L. Lanzerotti (Be11 Laboratories, Murray Hill, NJ, USA) began with a review of current work concerning the erosion of ices by energetic particle sputtering. After describing the experimental procedures used by his group for sputtering experiments, he presented recent laboratory data on sputtering of both water and sulfur dioxide ices. Sputtering yields (molecules ejected per incident ion) for these materials bombarded by high-energy ions in the range of several kilovolts to several MeV have been found to be much greater than predicted by traditional collision-cascade models. Theoretical understanding of this behavior is not complete, but models involving "ion explosion" mechanisms are among those being investigated. Experimenta1 results indicate that yields are proportional to stopping power in the material for light ions $\left(\mathrm{H}^{+}\right.$, He$\left.{ }^{+}\right)$, or approximately proportional to $(\mathrm{dE} / \mathrm{dx})^{2}$. Yields from higher mass ions such as $\mathrm{Fl}^{+}$, measured by the Caltech group (Trombrello, Haff and Watson) suggest even higher yields, but the stopping power for higher mass lons may not be well known. Other experiments by the Bell Labs group have demonstrated a temperature dependence to sputtering yields. For $\mathrm{H}_{2} \mathrm{O}$ ice, yields are temperature independent up to temperatures of about $110 \mathrm{~K}$ after which erosion increases significantly, with evidence that $O H, H$ and $O$ begin to come off at this point in addition to molecular $\mathrm{H}_{2} \mathrm{O}$. Similar results have been obtained for $\mathrm{SO}_{2}$, with somewhat lower critical temperature.

The net result of such sputtering depends on the nature of the ions impacting a surface, the target material, the size of the planet being bombarded and the energy spectrum of the eroded species (which is poorly known for the most part). For small, interplanetary ice grains, water ice will be eroded by solar wind sputtering at rates greater than sublimation at heliocentric distances greater than $\sim 1.5 \mathrm{AU}$. For larger planets and satellites, a large proportion of sputtered material will return to the surface on an atmosphereless object, with only the highest velocity fragments escaping. For an object with an atmosphere, material may be sputtered from the surface and thermalized in the atmosphere. If the object is exposed to solar wind or a magnetospheric environment, sputtered material may be ionized and swept away from the body. For conditions typical for the Gallilean satellites, sputtering should dominate sublimation for local and/or global redistribution of material if the surfaces are exposed to energetic heavy ion $\left(0^{+}, S^{+}\right)$fluxes.

The effects of magnetospheric interactions on energetic particle populations were described by M. F. Thomsen (Los Alamos National Laboratory, Los Alamos, NM, USA). She pointed out that measurements of the particle populations can be used as probes of neutral material in the magnetosphere, of the magnetic and electric field topology, of dynamical processes within the magnetosphere, and of sources and sinks of charged particles. Examples of the signatures left by the interaction of charged particles with satellites and with ring material were discussed. The characteristic signatures of such interactions are local minima in the particle fluxes. Both the broad, "macro," absorption signatures and the "micro" structure left by the most recent encounter between the particles and the satellite can be used to study absorption and deceleration processes and have also been used at Saturn to discover previously unknown satellite bodies and determine their orbital characteristics. The effects of Titan and E-ring material on the electron energy distributions were also described.

Charged particle populations may be used to complement and refine models of general magnetic and electric fleld topology. For example, recent fits to saturnian satellite particle absorption data constrain magnetic dipole parameters. Finally, Thomsen discussed the use of particle data to obtain dynamical information, such as the radial diffusion coefficients for energetic particles.

C. T. Russe11 (University of California, Los Angeles, CA, USA) discussed features of Titan's interaction with the saturnian magnetosphere and examined the analogous aspects of Venus' interaction with the solar wind. He pointed out that in the process of thinking through the comparison, more was learned about both situations than by studying each independently. Analysis of the magnetic field data around the Titan encounter suggests that the "wake" 
region was aberrated from the co-rotational direction by about $30^{\circ}$, with the magnetic field lines "bent" so strongly during the interaction that the field pointed along the wake in places. The case for Venus is not as clear, but the case presented was consistent with an aberration of $\sim 10^{\circ}$ and the existence of regions where the wake field is opposed to the interplanetary field. The field geometries at both Titan and Venus seem appropriate for magnetic field reconnection to occur in the wake.

L. J. Lanzerotti (Bell Laboratories, Murray Hill, NJ, USA) next reviewed some aspects of the Voyager energetic particle data near Saturn's magnetopause. The conditions near the magnetopause during the Voyager 2 encounter were different from those during the Voyager 1 . encounter at equivalent distances from Saturn, suggesting variable conditions at least in the outer part of the magnetosphere.

To close the session, T. V. Johnson (Jet Propulsion Laboratory, Pasadena, CA, USA) reviewed current knowledge about the effects of magnetospheric plasmas on satellite surfaces. He began by pointing out that the history of charged particle/surface interactions goes back to speculations about solar wind "darkening" of lunar material. Despite nearly two decades of work, however, firm evidence of major physical or optical effects is still lacking, although chemical changes and ion implantation have been studied extensively in lunar solids and meteorites. He reviewed current ideas about possible plasma/surface effects on the galilean satellites, including the possible role of sputtering on Io and spectral modification of Europa's surface by sulfur ions from Io. In the saturnian system, one of the issues under investigation is the role of the inner satellites as sources of heavy ions, particularly oxygen; Tethys and Dione appear to be strong sources, while Enceladus is not. Sputtering effects also limit the lifetime of micron size E-ring particles to $10^{3}-10^{4}$ years or less, requiring a source to replace these particles. In conclusion, Johnson noted that, while strong modification of satellite surfaces by plasma interactions has not yet been detected by remote sensing, Europa appears to be a good candidate for such effects, and local redistribution of ices by sputtering probably occurs on most satellites of the outer planet.

\section{PLASMA PHYSICS OF THE OUTER PLANETS}

Two sessions were devoted to discussion of plasma physics at Jupiter and Saturn. The principal topic of the first session was radio emissions from the planets. J. K. Alexander* (NASA Goddard Space Flight Center, Greenbelt, MD, USA) and A. Lecacheux (Observatoire de Meudon, Meudon, France), in separate papers, reviewed the observational results on the lowfrequency emissions from Jupiter and Saturn, with emphasis on phenomena first discovered in observations from Voyager, in particular the kilometric emissions (both narrow- and broadband) from Jupiter and from Saturn. There is evidence that kilometric broad-band emissions from both planets may originate from a source above the dayside high-latitude ionosphere, while the narrow-band kilometric emissions from Jupiter are generally believed to originate in the outer regions of the Io plasma torus and to reflect in their period (which is a few minutes longer than that of Jupiter's rotation) the well-known partial corotation of plasma in that region. Detailed observations of the frequency-time structure of jovian decametric emissions are now available but whether they represent source characteristics or arise from propagation effects remains a controversial question. I. de Pater* (University of Arizona, Tucson, AZ, USA) reviewed observations of the decimetric emissions produced by synchrotron radiation of energetic electrons in the inner jovian magnetosphere and the comparison with detailed model calculations that include electron energy spectrum, transport, loss, and the effect of nondipolar magnetic field components as well as inner satellites and the ring. R. Pellat* 3/ (Ecole Polytechnique, Palaiseau, France) discussed the theory of jovian decametric emissions, with emphasis on analogy to the terrestrial kilometric radiation. After providing a critical review of proposed mechanisms, he concluded that a generation mechanism based on a relativistic synchrotron maser instability appears the best candidate to explain the emissions.

Besides radio emissions, the topics of magnetospheric ion injection into the solar wind upstream of Jupiter and of magnetic field structure in the jovian magnetotail were each the subject of a paper. D. N. Baker* (Los Alamos National Laboratory, Los Alamos, NM, USA) and coworkers studied the observations by Voyager of energetic ions upstream of the jovian bow shock and concluded that, in contrast to the case of Earth where most such ions are thought to be locally accelerated at the bow shock, at Jupiter a significant direct injection from the magnetosphere takes place, as evidenced by the detection of a heavy mass component that can only have originated from the Io torus; they speculate that the dawn magnetopause may be a favored region for injection. K. W. Behannon (NASA Goddard Space Flight Center, Laboratory for Extraterrestrial Physics, Greenbelt, MD, USA) reported that Voyagers 1 and 2 found the detailed magnetic structure near and within the jovian magnetotail current sheet to be variable both with time and distance from Jupiter. The magnetic signature observed in that region was generally one of four types. These ranged from that of a static neutral sheet 
geometry to forms consistent with magnetic loop and/or $\mathrm{X}$-line geometry and possibly tearing mode Instability occurrence. These signatures are all similar to patterns found at various times at the earth's neutral sheet. Finally, a paper by E. M. Shoemaker (U. S. Geological Survey, Flagstaff, AZ, USA) on "Thermal evolution of Ganymede and Callisto" was rescheduled from an earlier session. Although not directly related to the topic of the session, his inferences from the age distribution of craters do have some implications for the magnetosphere of Jupiter; the loss of surface material from these satellites by sputtering must have amounted to no more than some tens of meters over their age, which is consistent with present idea on the sputtering rate and the flux of charged particles.

In the initial talk of the second session on plasma physics of the outer planets, D. A. Gurnett (University of Iowa, Iowa City, IA, USA) illustrated convincingly the scientific value of comparative magnetospheric studies. He summarized the various types of plasma waves observed by the Voyager spacecraft at Jupiter and Saturn and noted terrestrial analogies. He emphasized that there are close similarities in terms of the types of plasma wave phenomena involved, but also marked differences resulting from differences in large-scale structure of the two planets. The decametric radiation at Jupiter and kilometric radiation at Saturn provide examples, apparently being produced in the same plasma wave mode by the same mechanism but in different magnetospheric regions under different physical conditions (foot of the Io torus vs. the polar cusp at Saturn). At Jupiter, the low-density magnetospheric cavity is filled with intense trapped continuum radiation at frequencles above the electron plasma frequency. Such radiation was not found in the initial analysis of data from Saturn, but the results of a more careful analysis show that it is present at Saturn also, with low intensity as at Earth. It has been found further that the trapped, so-called continuum radiation originates in fact as narrow-band emissions. Multiple reflections at the moving magnetopause introduce Doppler-shift and spreads the radiation into a continuum. Similar narrow-band emissions have also been found in terrestrial continuum emissions. They appear to be generated at highly localized regions of the plasmapause. The source regions at Jupiter and Saturn may also be localized regions where there are strong gradients in plasma density.

The latest results on Saturn's magnetic field and magnetosphere were summarized by $M$. $H$. Acuña (NASA Goddard Space Flight Center, Greenbelt, MD, USA) and coworkers. He reviewed the major findings by Voyager 1: the confirmation of a predominantly dipolar main field of $<1^{\circ}$ tilt, the presence of a significant azimuthal ring current, the existence of a magnetotail, and the discovery of an induced magnetosphere with bipolar tail at Titan, formed in its interaction with the corotating saturnian magnetospheric plasma. Problems concerning the determination of the internal magnetic field of Saturn were discussed in detail, the major question being whether or not there is a northward offset of the dipole as implied by charged particle absorption results. It was shown that the field model most recently developed on the basis of Voyager 2 observations, a 3-term, zonal harmonic coefficient (23) model, gives consistency among all observations (V1, V2, P11). In this mode1, the octupole coefficient is larger than the quadrupole term. Particle absorption predictions cannot distinguish between the zonal and offset dipole models. Nothing has been found in the magnetic field observations that could account for the periodic modulation of saturnian kilometric radiation (SKR).

A. J. Dessler (Rice University, Houston, TX, USA) discussed his theory of corotating magnetospheric convection at Jupiter. A large-scale convection pattern driven by the solar wind exists at Earth. At Jupiter the convection pattern would be driven internally, with the pattern of convective flow lines corotating with the planet. The principal source of plasma for this convective transport would be the Io plasma torus. The physics of such a system bears similarities to that proposed for pulsars. The power available from the internal plasma source and the rapldly rotating magnetosphere is extremely large. Dessler argues that the concept of corotating convection can provide a means of unifying apparently disparate jovian phenomena.

The results of calculations on the force balance in the magnetospheres of Jupiter and Saturn were presented by $R$. L. McNutt* (Massachusetts Institute of Technology, Cambridge. MA, USA). Specifically, radial force-balance in the middle magnetospheres of the two planets was considered. The results suggest that at Saturn the Lorentz force balances the centrifugal force of the cold corotating plasma. At Jupiter, however, the centrifugal force balances only $\sim 25$ percent of the Lorentz force, with the remafning part balanced by pressure gradients in the hot, high $B$ plasma of the magnetodisk.

V. M. Vasyliunas (Max Planck Institut für Aeronomie, Katlenburg-Lindau, FRG) discussed thin current sheets in planetary magnetospheres and the validity and applicability of two different approximations for modeling them. He pointed out that extended sheets of plasma and electric current are a prominent feature of the magnet8spheres of the giant planets, particularly Jupiter. He asserted that the variations of the magnetic field with radial distance from Jupiter observed outbound by $\mathrm{P} 11, \mathrm{~V} 1$ and $\mathrm{V} 2$ are consistent with no significant local time differences out to $100 \mathrm{R}_{\mathrm{J}}$ (an interpretation with which the Voyager magnetometer investigators disagree). In contrast, he stated, there is a very pronounced local time difference in Earth's magnetosphere. He further interpreted the observations as showing that the 
measured $B_{\phi}$ component of the field is simply that needed to maintain the plasma in corotation. From these arguments and others not discussed in his presentation, he concluded that the jovian magnetotail begins only at a distance of $100 \mathrm{R}_{\mathrm{J}}$ or greater.

The observations related to the control of jovian decametric radiation (DAM) by Io were reviewed by C. K. Goertz* (Max-Planck Institut für Aeronomie, Katlenburg-Lindau, FRG). He then provided his theoretical interpretation of the observations, using geometrical arguments based on a conical sheet model of the sources. He argued that within the longitude sector $\phi \sim 200 \pm 90^{\circ}$, Io excited Alfvén waves which are then confined to the region north of the Io torus (a suggestion challenged in the discussion that followed). Multiple Alfvén waves would produce the multiple arcs seen in the Voyager radio astronomy data. Parallel electric fields would accompany the Alfvén wave pulses; at high latitudes, electrons could be accelerated to energies $>100 \mathrm{eV}$, further exciting ion-cyclotron and upper and lower hybrid electrostatic waves, all of which may play crucial roles in generating DAM.

\section{RINGS: DYNAMICS AND ELECTRODYNAMICS}

Although the final session of the Symposium was the only one to deal specifically with planetary rings, ring-related science had been a frequent topic of discussion throughout the week. of the seven papers presented, only three were devoted to new observations and most of the time went to discussions of analysis and development of theory necessary to interpret the data. A. F. Cook* (Herzberg Institute of Astrophysics, Ottawa, Canada) led off with an overview that traced the development of (currently relevant) ideas about rings from Laplace in 1802 up to the present. E. C. Stone (Ca1ifornia Institute of Technology, Pasadena, CA, USA), this time filling in for G. L. Tyler (Stanford University, Stanford, CA, USA) and coworkers, presented the results obtalned from the Voyager 1 radio occultation by the rings of Saturn. Data were obtained at two wavelengths, 3.6 and $13 \mathrm{~cm}$. Together, the two measurements yield information on the particle size distribution. Characteristic maximum particle diameters are 11, 10 and $2 \mathrm{~m}$ in ring $A$, the Cassini division and ring $C$, respectively. The characteristic minimum particle diameters are highly variable and range from less than one cm up to several $\mathrm{cm}$.

Other features of ring structure were described by R. A. West, speaking for L. W. Esposito (University of Colorado, Boulder, $\mathrm{CO}$, USA) who reviewed the Voyager photopolarimeter observations of a star occulted by the saturnian rings, measurements which provided ring data at the highest spatial resolution. The chief new results presented in ottawa were (1) the mass of the rings is about $10^{-7}$ of the mass of Saturn, and (2) the surface or areal mass density of the rings is about $100 \mathrm{~g} \mathrm{~cm}^{-2}$ in the regions where the optical depth is unity.

Several papers explored the consequences of ambient plasma and electromagnetic forces for ring structure and for spoke formation. In particular, D. A. Mendis* (Iniversity of California, San Diego, CA, USA) and H. Alfven discussed the role of "gravito-electrodynamics" in the explanation of various ring effects. Mendis and Alfvén believe that the saturnian ring system represents a "time-capsule" that can provide clues about physical processes operating during the early stages of its formation and, by extension, during early stages of evolution of the solar nebula.

The impulsive radio discharge data in the frequency range of $20 \mathrm{kHz}$ to $40 \mathrm{MHz}$ was discussed by J. W. Warwick* 4/ (University of Colorado, Boulder, CO, USA). He suggested that these bursts may have originated from static electric discharges in the rings, although other ringrelated source mechanisms may also be possible. In Warwick's picture, charged dust grains, accelerated in the discharges, form the spokes. Others present pointed out that lightning storms in the planet's equatorial region may explain the discharge, a possibility that also needs further study.

The electromagnetic effects on Saturn's rings were also discussed by G. Morfil1* (Max-P1anck Institut für Kernphysik, Heidelberg, FRG). Calculations were presented showing that the ring particle spacing is less than the Debye length for the expected plasma conditions. Therefore, Morfill takes issue with Warwlck's model of charged dust grains, stating that the individual ring particles will not become charged. The ring as a whole, however, is acted upon by the photoelectric effect and attains a potential of about $+10 \mathrm{~V}$. Next, Morfill focused attention on the "spokes" in the B-ring. He proposed that these can be understood in terms of the plasma produced by impacts of clusters of micrometeoroids on ring particles. Such an event of suitable size would produce locally an enhanced density of plasma within which small dust particles could become negatively charged and rise above larger particles of higher inertia. As the saturnian magnetic field penetrates the plasma, the torentz force induces an electric polarization. The plasma then rapidly moves radially outward (or inward if the impacts occur at less than the corotation distance from Saturn). Along the way, 
additional dust grains are charged until the plasma finally becomes dispersed. Furthermore, Morfill suggests that the frequency of spoke occurrence is in agreement with the expected meteoroid flux, giving additional weight to this suggested mechanism.

The meteoroid flux, important to Morfill's arguments, had been discussed earlier in the session, but only for Jupiter's environment. S. Y. Su (Lockheed-Emsco, Inc., Houston, TX, USA) and $\mathrm{H}$. A. Zook stated that none of the potential particle sources in the jovian system, including those from a variety of hypothetical sateliftes, can supply the observed count rates of the Pioneer 10 meteoroid penetration experiment. Of particular difficulty is matching the factor of ten difference in count rate between the inbound and outbound legs of the encounter. However, a suitable low-velocity source external to the fovian system could explain the data.

\section{SUMMARY}

The four days of meetings offered participants a remarkably informative review of the current state of knowledge and the promising directions for further inquiry in studies of the outer planets and their environments. This review has attempted to include the ideas that the authors found particularly engrossing but a summary is necessarily selective. We urge our readers to inform themselves further about the geophysics of the outer planets by reading the papers that follow in this volume and in accounts of current work in the standard geophysical journals.

\section{ACKNOWLEDGEMENTS}

This work was supported in part by funds from the National Aeronautics and Space Administration (USA) under grant NGL 05-007-004. 